\title{
Clusters for Cosmology
}

\section{Alain Blanchard*}

Institut de recherche en astrophysique et planétologie

E-mail: alain.blanchardeirap.omp.eu

The properties of clusters are known to provide critical information of cosmological relevance. I will discussed the various observationnal probes on clusters and how physical models of clusters can be build based on simple scaling laws. I will show haw the standard predictions of these models within the concordance picture conflict with present day observations, leading to the cluster conendrum: $\mathrm{x}$-ray temperature distribution of clusters at different redshift conflicts with standard scaling expectations, SZ clusters counts as obtained by PLANCK lead to cosmological parameters in tension with those obtained from the CMB fluctuations. Solutions to evade this problem will be discussed as well as ways to progress on this issue.

References

[1] Blanchard, A., Evidence for the fifth element. Astrophysical status of dark energy, A\&A Rev., 18, 595 (2010)

[2] Blanchard, A., \& Douspis, M., Evidence for new physics from clusters? , A\&A 436, 411 (2005)

[3] Linder, E. V., Cosmology with x-ray cluster baryons, JCAP 4, 4 (2007)

Frontiers of Fundamental Physics 14 - FFP14,

15-18 July 2014

Aix Marseille University (AMU) Saint-Charles Campus, Marseille

${ }^{*}$ Speaker. 